Revista Universo Contábil, ISSN 1809-3337

FURB, v. 6, n.1, p. 156-170, jan./mar., 2010

doi:10.4270/ruc.2010109

Disponível em www.furb.br/universocontabil

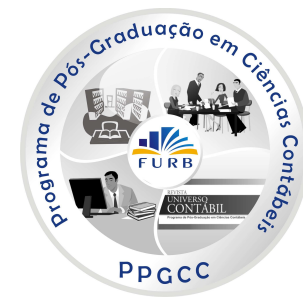

\title{
CONVERGENCIA INTERNACIONAL EN MATERIA DE SUBVENCIONES: REFERENCIA A LOS DERECHOS DE EMISIÓN DE GEI*
}

\section{INTERNATIONAL CONVERGENCE OF GOVERMENT GRANTS: A STUDY OF GHG EMISSION RIGHTS +}

\author{
Ana Isabel Mateos Ansótegui \\ Doctora en Economía pela Universidad CEU Cardenal Herrera \\ Profesora do Departamento de Economía y Empresa \\ Universidad CEU Cardenal Herrera \\ Dirección: C/ Carmelitas 3 \\ CEP: 03203 - Elche - España \\ E-mail: amateos@uch.ceu.es \\ Teléfono: 0034965426486
}

\section{RESUMEN}

Es indudable la necesidad de una información financiera comparable a nivel internacional en un mercado de capitales global. Dicha información debe ser elaborada bajo unos estándares de alta calidad para que cumplan su objetivo, siendo la participación del IASB y el FASB clave en dicha elaboración. Desde los acuerdos de Norwalk en 2002, FASB e IASB están coordinando sus agendas de trabajo y desarrollando proyectos conjuntos para la elaboración de unos estándares internacionales de alta calidad. El objetivo del presente trabajo es presentar dicho proceso de convergencia, y en particular la situación de la NIC 20 sobre Subvenciones dada la trascendencia de ésta en el registro contable de los Derechos de Emisión de Gases de Efecto Invernadero. En particular, y respecto al tema que nos ocupa, se necesita una revisión en profundidad de la NIC 20 sobre Subvenciones para eliminar las inconsistencias actuales con el Marco Conceptual y así unificar criterios para conseguir que las empresas que participan en los diferentes Esquemas de Comercio de Derechos de Emisión elaboren estados financieros comparables y útiles en los procesos de decisión empresarial.

Palabras clave: Convergencia internacional. Subvenciones. Derechos de emisión. Protocolo de Kioto.

\footnotetext{
Artigo recebido em 05.09.2009. Revisado por pares em 27.10.2009. Reformulado em 02.12.2009. Recomendado em 09.12.2009 por Ilse Maria Beuren (Editora). Publicado em 02.10.2010. Organização responsável pelo periódico: FURB.

Este trabajo se enmarca en el proyecto de investigación "Tributación del comercio de derechos de emisión y su problemática legal y contable". Entidad financiadora: BSCH. Proyecto precompetitivo USP-BSCH-04/08.
} 


\section{RESUMO}

É incontestável a necessidade de uma informação financeira comparável ao nível internacional em um mercado de capitais global. Tal informação deve ser elaborada com base em padrões de alta qualidade para que cumpra seu objetivo, sendo a participação do IASB e do FASB fundamental em tal elaboração. Desde os acordos de Norwalk em 2002, o FASB e o IASB estão coordenando suas agendas de trabalho e desenvolvendo projetos conjuntos para a elaboração de padrões internacionais de alta qualidade. O objetivo deste estudo é apresentar o processo de convergência, e em particular a situação da NIC 20 sobre Subvenções, dada à transcendência desta no registro contábil dos Direitos de Emissão de Gases de Efeito Estufa. Em particular, a respeito do tema em questão, se necessita de uma revisão em profundidade da NIC 20 sobre Subvenções para eliminar as inconsistências atuais com o marco conceitual e assim unificar critérios para conseguir que as empresas que participam nos diferentes esquemas de Comércio de Direitos de Emissão elaborem demonstrações financeiras comparáveis e úteis ao processo de decisão empresarial.

Palavras-chave: Convergência internacional. Subvenções. Direitos de emissão. Protocolo de Quioto.

\section{ABSTRACT}

The necessity of comparable financial information at international level in a global market of capitals is doubtless. Such information must be elaborated under high quality standards in order to achieve its objective, being essential the IASB and FASB participation in the elaboration. Since Norwalk Agreement in 2002, FASB and IASB are conducting new projects to elaborate high quality international accounting standards. The objective of this paper is to show the convergence process, in particular the state of IAS 20 Government Grants and the accounting of GHG Emission Rights. A depth revision of IAS 20 is needed in order to eliminate inconsistencies with the Conceptual Framework and to unify criteria that allow us to obtain comparable and useful financial statements form those companies that participate in different Schemes of Tradable Permits.

Keywords: International convergence. Goverment grants. Emission rights. Kioto Protocol.

\section{INTRODUCCIÓN - EL PROCESO DE ARMONIZACIÓN INTERNACIONAL}

En estos momentos estamos siendo testigos de un paso más hacia la convergencia internacional contable. La publicación de la actualización del MoU (Memorandum of Understanding) firmado entre el International Accounting Standard Board (IASB) y el Financial Accounting Standard Board (FASB) el 11 de septiembre de 2008 establece los objetivos a alcanzar antes de 2011 en los principales proyectos conjuntos en temas como Consolidación, Instrumentos Financieros, Arrendamientos, etc. Ello unido a la posibilidad de que algunas empresas estadounidenses puedan presentar sus estados contables elaborados según Normas Internacionales Información Financiera (NIIF) para cumplir con sus obligaciones frente a la Securities and Exchange Commission (SEC) para los ejercicios fiscales terminados el 15 de diciembre de 2009, podría traducirse en una obligatoriedad del uso del las normas emitidas por el IASB en 2014.

Así, los dos grandes emisores de estándares contables a nivel mundial (IASB y FASB) mantienen continuos contactos para acercar posturas, de forma que las normas de ambos organismos están siendo objeto de continua revisión. El presente trabajo se centra en la situación de la IAS 20 del IASB Contabilización de las subvenciones oficiales y presentación 
de las ayudas oficiales desde una doble perspectiva: la adaptación de la misma a nuestro PGC2007 en la Norma de Registro y Valoración $18^{\circ}$ Subvenciones, Donaciones y Legados recibidos y la posible convergencia con la SFAS 116 Accounting for Contributions Received and Contributions Made del FASB, tomando como referencia el caso de los Derechos de Emisión de Gases de Efecto Invernadero. En efecto, el IASB ha dejado en suspenso la emisión de una norma de registro de los mismos en tanto en cuanto - dada la estrecha relación entre ambos temas - no esté finalizado el proyecto sobre la IAS 20.

Este proceso de convergencia y armonización contable internacional tiene su origen primeros acuerdos de 1995 entre el entonces International Accounting Standard Comité (IASC) - hoy IASB - y el Organización Internacional de los Organismos Rectores de las Bolsas (IOSCO). Estos acuerdos culminaron el 17 de mayo de 2000 en la recomendación de la IOSCO a todos sus países miembros de que utilizaran las Normas Internacionales de Contabilidad emitidas por el IASB. Así, las empresas europeas veían más cerca la posibilidad de contar con un cuerpo normativo único y estandarizado que fuera aceptado por cualquier bolsa en la que cotizaran. En concreto, abriría la posibilidad a que las empresas extranjeras que cotizaran en Estados Unidos no tuvieran que presentar los correspondientes documentos de conciliación con los US GAAP de ingresos y activos netos que la SEC les exigía hasta ese momento.

Ha de recordarse además que el acuerdo inicial entre el IASC y el IOSCO recibió un apoyo crucial cuando la Securities and Exchange Comisión (SEC) (http://www.sec.gov/news/studies/acctgsp.htm), en un comunicado de prensa de abril de 1996, manifestó su disposición a aceptar las NIC resultantes de esta revisión siempre y cuando estas satisficieran tres principios:

(i) Los estándares deben incluir un conjunto básico de pronunciamientos contables que supongan un completo y generalmente aceptado fundamento de la contabilidad.

(ii) Los estándares deben ser de alta calidad - deben suponer una mejora en la comparación y transparencia - y exigir información completa. Los inversores deben ser capaces de analizar la evolución de la gestión en el tiempo y entre las distintas compañías.

(iii) Los estándares deben ser interpretados y aplicados rigurosamente. Si los estándares contables deben satisfacer el objetivo de contabilizar operaciones y hechos similares de la misma forma - con independencia de quien los aplique o donde sean aplicados -, auditores y organismos reguladores deben insistir en una rigurosa aplicación de estos estándares. De otra manera no se conseguiría el objetivo de facilitar la comparación y la transparencia.

Pero antes de poder llegar a ese punto de armonización contable internacional real, mucho camino quedaba por andar. En noviembre de 1995, la Unión Europea, a la vista de la inoperatividad de sus propias Directivas para lograr la comparabilidad de las Cuentas Anuales de las empresas europeas (COMISIÓN EUROPEA, 1995), decide cambiar su estrategia, y aceptar las normas ya emitidas por el IASB como un marco normativo aplicable a las empresas europeas. En un comunicado en el año (COMISIÓN EUROPEA, 2000), ya se sientan las bases para la promulgación del Reglamento (COMISIÓN EUROPEA) n. 1606/2002 de 19 de julio de 2002 del Parlamento Europeo y del Consejo que obliga, ex art. 4, a las empresas cotizadas en cualquier bolsa europea que pertenezcan a un grupo, a presentar sus Cuentas Anuales Consolidadas a partir de el $1^{\circ}$ de enero de 2005 de acuerdo con las Normas Internacionales Información Financiera (NIIF) - antes llamadas Normas de Internacionales de Contabilidad (NIC) - elaboradas por el IASB. 
La postura de claro liderazgo del IASB en Europa en cuanto a emisora de normativa contable internacional, se ve potenciada con la decisión tomada el 18 de septiembre de 2002 entre el IASB y el FASB de trabajar conjuntamente en la convergencia de las normas a nivel mundial, acuerdo que se vendría a conocer por el nombre de Acuerdo Norwalk, por la ciudad donde tuvo lugar dicho encuentro. Ambos organismos establecieron dos compromisos básicos: a) desarrollar normas de alta calidad, que sean compatibles entre si; y b) eliminar las diferencias necesarias entre IFRS y los Principios de Contabilidad Generalmente Aceptados en los Estados Unidos (US GAAP), garantizando que la comparabilidad de las normas se mantengan en el tiempo.

Desde 1973, el FASB ha sido el organismo del sector privado designado para el establecimiento de los principios de información y contabilidad financiera en los Estados Unidos de América. Están oficialmente reconocidos por la Securities and Exchange Commission (SEC) (Financial Reporting Release $n^{\circ}$. 1, Section 101) y el American Institute of Certified Public Accountants (AICPA) (Rule 203, Rules of Professional Conduct, as amended May 1973 and May 1979). Su principal misión es proveer de unos estándares contables para la guía y educación del público en general, y los auditores y usuarios de la información financiera en particular.

Fruto de este acuerdo, el 27 de febrero de 2006, ambos organismos publicaron la primera versión del antes mencionado Memorandum of Understanding (http://www.iasplus.com/pressrel/0602roadmapmou.pdf), donde se recogen las condiciones y fechas para que pueda darse ese último paso en aras de una armonización internacional real. Así, la eliminación del requisito de conciliación exigido por la SEC antes citado, sería posible a partir del $1^{\circ}$ de enero de 2009 si las NIIF se aplican de manera efectiva por un gran número de empresas en un gran número de países y se logra un apreciable avance en el proyecto de convergencia desarrollado conjuntamente por FASB e IASB (SEC - Proposed Rule 33-8818 Acceptance from Foreign Private Issuers of Financial Statements Preparedin Accordance with International Financial Reporting Standards without Reconciliation to U.S. GAAP).

Ahora bien, como indica García-Ayuso Covarsí (2006):

Aunque la convergencia entre el modelo contable del IASB y el del FASB pueda verse como algo deseable y cualquier esfuerzo tendente a lograrla pueda considerarse, en principio, encomiable, puede no ser razonable apoyar el acercamiento de los dos marcos normativos a toda costa.

Como ya expresó Sir David Tweedie, presidente de IASB (STREET, 2002):

Estamos tratando de fusionar ambos cuerpos normativos, y dejar de tener USGAAP o NIC. Pero por muchos años las diferencias continuarán. Tan pronto como sea posible, debemos trabajar para resolver estas diferencias significativas a fin de dejar diferencias triviales. Las principales diferencias deben eliminarse, para que desaparezca el asunto de la conciliación [de estados financieros]. En algunos casos las normas de Estados Unidos cambiarán, y en otras las NIC cambiarán. Actualmente es muy difícil decirle a las compañías estadounidenses que todo el mundo utiliza NIC, pero que ellos pueden utilizar US GAAP. Esto es una fuente continua de fricción. La clave es hacer que el asunto de US GAAP versus NIC empiece a desaparecer y en su lugar acercar ambos pronunciamientos.

En efecto, mientras que el modelo del IASB es un modelo basado en principios, como posteriormente se comentará, el modelo estadounidense está basado en reglas. Se trata por tanto de hacer converger dos esquemas radicalmente distintos, lo que supondrá que uno u otro tendrá que hacer cambios en sus pilares básicos. Y esa renuncia no parece fácil. 


\section{EL IASB}

\subsection{Funcionamiento}

El IASB, con sede en Londres, comienza sus actividades en 2001, tras la aprobación de la nueva Constitución del IASC como Fundación (IASCF). El IASC se creó en 1973 por las organizaciones profesionales de 9 países - Australia, México, Japón, Estados Unidos, Canadá, Alemania, Francia, Holanda y Reino Unido - en respuesta a las necesidades de armonización contable manifestadas por numerosas empresas, instituciones financieras, bancos, etc. que tras más de 25 años de intensa actividad se ha convertido en emisor de normas contables internacionalmente reconocidas, siendo su objetivo principal publicar estándares contables internacionales que permitan una información financiera transparente y comparable en los estados financieros.

Respecto a la denominación de las normas, cabe señalar que las normas emitidas por antiguo IASC se llamaban NIC, y sus interpretaciones SIC. Tras el cambio de estructura del organismo emisor, las normas publicadas a partir de ese momento pasan a llamarse NIIF y sus interpretaciones IFRIC. La numeración no ha seguido correlativa, sino que tanto para las NIIF como las IFRIC han comenzado desde el número 1. En la línea del propio IASB, el acrónimo NIIF debe usarse en sentido genérico, esto es, para indicar tanto una antigua norma (NIC) como una nueva (NIIF) en el marco del conjunto de normas internacionales, si bien para referirnos a cada una de ellas, se mantiene en nombre y numeración antiguo (http://www.iasplus.com/standard/standard.htm).

Es necesario precisar, atendiendo al art. 2 del Reglamento (COMISIÓN EUROPEA) n. 1606/2002 del Parlamento Europeo y del Consejo, qué se entiende exactamente por Normas Internacionales de Contabilidad. En concreto, cabe resaltar que el citado precepto establece que las normas internacionales de contabilidad cabe identificarlas con la suma de las Normas Internacionales de Contabilidad (NIC), las Normas Internacionales de Información Financiera (NIIF) y las interpretaciones correspondientes (interpretaciones del SIC/interpretaciones del IFRIC), las modificaciones ulteriores de dichas normas y de las interpretaciones correspondientes, así como las futuras normas y las interpretaciones correspondientes que pueda elaborar o aprobar el International Financial Reporting Interpretations Committe (IFRIC).

Funcionando desde marzo de 2002, era antes conocido por Comité de Interpretación de los Standards (SIC). Este comité fue creado en 1997 para asegurar la aplicación rigurosa y la correcta comparabilidad a nivel internacional de los estados financieros. Las propuestas de interpretación o borradores (Draft Interpretations) son expuestas a comentario público en la web del IASB durante 90 o 120 días. El objetivo es recoger los comentarios de organismos contables (públicos y privados) de todo el mundo, y, tras su debate, ser aprobadas por el IFRIC y enviadas al IASB para su revisión y aprobación como NIIF. Sus interpretaciones son llamadas como el organismo que las emite, IFRIC, aunque también se hace referencia a ellas como Final Interpretations, numeradas correlativamente y de forma independiente a las antiguas interpretaciones llamadas SIC.

En materia de NIIF, nos encontramos, por tanto, ante normas que requieren ser desarrolladas e interpretadas a efectos de asegurar su correcta aplicación así como la comparabilidad de los estados financieros de las empresas en el plano internacional. El proceso de aceptación de las normas (endorsment) establecido en el Reglamento 1606/2002 señala que dicho mecanismo opera en dos niveles: a través del consejo dado por el European Financial Reporting Advisory Group (EFRAG) a la Comisión y a través de la consulta que la Comisión realiza al Comité de Reglamentación Contable (Accounting Regulatory Comité - 
ARC). El ARC está integrado por representantes de los Estados miembros y presidido por un representante de la Comisión.

El EFRAG (http://www.efrag.org) es un organismo consultor, privado e independiente, de la Comisión Europea en materia de normativa contable internacional. Se creó en 2001 con una estructura de dos cuerpos. El organismo supervisor y el grupo técnico. Éste asesora a la Comisión Europea antes de adoptar cualquier norma contable internacional o cualquier interpretación. Determina si una NIIF o interpretación responde a la imagen fiel (principio establecido por las Directivas IV y VII) y, además, si de ella se derivará información fiable, relevante, comprensible y comparable. Así mismo, participa en el proceso de discusión previo de elaboración de la normativa por el IASB, valorando los documentos de trabajo o los borradores que se van editando.

La última consulta tiene por objetivo asegurar que favorezca el interés público europeo, el cual se deja al juicio de la Comisión, dado su carácter político y no técnico. Para que el proceso se complete deben traducirse las normas a los idiomas de todos los países de la UE, y finalmente, si son aceptadas, serán publicadas en el Diario Oficial de la Unión Europea (GINER-INCHAUSTI, 2004) así como en el IASB's Annual Bound Volume of Standards.

Se ha de tener en cuenta que no todas las NIIF son obligatorias en el ámbito europeo, si no sólo aquellas que hayan sido adoptadas por la Unión Europea. De hecho, la Comisión adopta normas internacionales de contabilidad mediante un procedimiento de comitología conforme al Reglamento (COMISIÓN EUROPEA) nº 1.606/2002 indicándose la posibilidad de dicha adopción en la medida en que su aplicación dé una visión cierta y equitativa de la situación financiera y de los resultados de una sociedad, favorezcan el interés público europeo y cumpla criterios básicos sobre la calidad de la información requerida para que los estados financieros sean útiles para los usuarios.

Comitología es un procedimiento por el que la Comisión Europea recurre para, la adopción de actos de ejecución de actos legislativos, a la asistencia de un Comité. Los Comités son foros de debate presididos por la Comisión y compuestos por representantes de los Estados miembros, que le permiten a la Comisión intercambiar opiniones con las administraciones nacionales antes de adoptar cualquier medida de ejecución. De este modo, la Comisión se asegura de que dichas medidas se adapten lo mejor posible a la realidad de cada país. Supone que las decisiones se toman por mayoría cualificada (y con votos ponderados).

El conjunto de NIIF que han sido adoptadas en la UE es recogido por los Reglamentos publicados a partir del Reglamento (COMISIÓN EUROPEA) $n^{\circ}$ 1.725/2003 de la Comisión de 29 de septiembre de 2003. Éste, en su apartado (5) indicaba que deberían adoptarse todas las NIC existentes a 14 de septiembre de 2002, a excepción de la NIC 32 - Instrumentos financieros: presentación e información a revelar, la NIC 39 - Instrumentos financieros: reconocimiento y valoración y las interpretaciones relacionadas con ellas. Las NIC 32 y 39 serían adoptadas posteriormente por los Reglamentos $n^{\circ} 2.086 / 2004$, de 19 de noviembre de 2004, y $\mathrm{n}^{\circ} 2.236 / 2004$, de 29 de diciembre de 2004, (http://ec.europa.eu/internal_market/accounting/ias_en.htm).

Debe hacerse hincapié en una característica básica de las NIIF antes señalada. Se trata de normas que se basan en un tratamiento de referencia (benchmark) y un tratamiento permitido (allowed). En claro contraste con el sistema contable español, basado en reglas, las normas internacionales describen para su aplicación en la empresa al menos dos posibilidades de registro y valoración: un tratamiento de referencia y uno alternativo. Estudios recientes han contabilizado más de cien alternativas a la hora de poner en práctica estas normas internacionales.

Entraremos en detalle sobre las implicaciones de diferentes tratamientos de una misma operación y la decisión tomada por el legislador español en materia de subvenciones en 
epígrafes posteriores.

\section{2 ¿Quién Aplica las NIIF?}

Más de 100 países han apostado por una convergencia de sus normas nacionales a los estándares del IASB, si bien el grado de aplicabilidad no es homogéneo en estos momentos. Se espera que se adopten también en Chile (2009), Corea (2009), Brasil (2010), India (2011) y Canadá (2011). Aún otros países, como China y Japón, si bien directamente no están adoptando los IFRS, han acordado trabajar con la International Accounting Standards Board (IASB) para eliminar para el 2011 las principales diferencias entre los principios de contabilidad generalmente aceptados del país sede $y$ las NIIF (http://www.iasplus.com/country/useias.htm).

No obstante, debemos subrayar que en el mencionado Reglamento núm. 1606/2002 se otorga a los distintos Estados miembros la capacidad de decidir la legislación que en esta materia le será aplicable tanto a sus empresas individuales como a los grupos no cotizados en mercados regulados. En este sentido, en la Comunicación 359 final (Comisión al Consejo y al Parlamento Europeo, COM 2000), de junio de 2000, pese a que la Comisión sostiene la inoperancia del uso de las NIC en las cuentas individuales debido a los requisitos reguladores y fiscales, recomienda a los Estados miembros que "en la medida de lo posible, [...] deberían fomentar el uso de las NIC, o incluso exigirlo, también para las cuentas individuales. Así, se facilitaría más adelante la preparación de cuentas consolidadas".

Ahora bien, seguirá siendo aplicable la normativa contable nacional a efectos de la confección de las Cuentas Anuales Individuales de todas las empresas (formen grupo o no) e, incluso, de las Cuentas Anuales Consolidadas respecto de los grupos de empresas no cotizados. Nos referimos a aquellos grupos de empresas, en que los valores de ninguna de las empresas que lo integran hayan sido admitidos a cotización en un mercado regulado de cualquier Estado miembro.

La recomendación anterior ha sido seguida por distintos países que han adoptado las NIIF para la elaboración de sus cuentas anuales individuales y, por tanto, para la cuantificación del resultado empresarial a efectos de la posterior liquidación del IS. En concreto, a comienzos de 2008 podemos señalar diversos países que han adoptado las NIIF como normativa contable nacional, siendo necesario distinguir distintas posibilidades:

a) Cuentas anuales individuales de empresas cotizadas

- Se obliga a su uso en: Bélgica (las empresas inmobiliarias estatales están obligadas; para el resto están pendientes de examinar las implicaciones fiscales y legales de su obligación); Bulgaria; Chipre; República Checa; Dinamarca (a partir de 2009); Estonia; Grecia; Italia (excepto para compañías aseguradoras); Letonia; Lituania; Malta; Portugal (sólo para bancos, compañías de seguros, y otras instituciones financieras); Eslovaquia (sólo para empresas de interés público - bancos, el Banco de Exportación-Importación de la República Eslovaca, aseguradoras y reaseguradoras excepto las sanitarias, Mercado de valores, Compañía Eslovaca de Ferrocarriles, etc); e Islandia.

- No se obliga, pero se permiten su uso en: Finlandia; Irlanda; Luxemburgo; Holanda; Eslovenia; Reino Unido; Liechtenstein; y Noruega.

b) Cuentas Anuales individuales de resto de empresas

- Se obliga su uso en: Bulgaria (excepto para PYMES, y empresas en liquidación o insolvencia); Chipre; Estonia; Italia (compañías financieras 
supervisoras; compañías con instrumentos financieros ampliamente distribuidos entre el público); Letonia (bancos, compañías de seguros, e instituciones de crédito); Estonia (bancos, compañías de seguros, e instituciones de crédito); Malta; Polonia; Eslovaquia.

- No se obliga, pero se permite su uso en (restringido por tipos de empresas): Dinamarca; Finlandia; Grecia; Irlanda; Holanda; Polonia; Portugal; Reino Unido; Liechtenstein; y Noruega.

Como puede comprobarse, estamos asistiendo a una implantación progresiva de las NIIF para todas las empresas comunitarias, de ahí la necesidad de la armonización discutida en párrafos precedentes. De esta forma, para aquellas empresas que ya aplican las NIIF, bien por obligación o bien por decisión propia, cuentan con la influencia determinante del Marco Conceptual del IASB, que ha de tomarse como referencia de trabajo básico. El Marco Conceptual del IASB es el la referencia obligada para la correcta interpretación de la normativa contable internacional, evitándose así interpretaciones absurdas o inconsecuentes.

\section{EI FASB}

El FASB (Financial Accounting Standards Board) es un organismo privado que tiene a su cargo la elaboración de los estándares para la presentación de los estados financieros en Estados Unidos. Fundado en 1973, con sede en Norwalk (Connecticut), consta de 5 miembros, asistidos por 68 profesionales de diferentes áreas. La elaboración de dichos estándares, los llamados US-GAAPS, es competencia del FASB por designación de la Securities and Exchange Commission (SEC) y el American Institute of Certified Public Accountants (AICPA) quien a través de la Rule 203 da carácter de normas de obligado cumplimiento a las emitidas por dicho organismo privado.

Tras el colapso bursátil de 1929, fue creado el Committee on Accounting Procedure (CAP) por parte del AICPA en 1938, que empezó a publicar una serie de boletines acerca de prácticas y normas contables. Sin autoridad para obligar a la puesta en práctica de sus procedimientos, en 1959 el AICPA creó un nuevo organismo con la intención de fomentar la elaboración de los principios contables generalmente aceptados, el Accounting Principles Board (APB), pero debido a las críticas recibidas sobre su funcionamiento a finales de los años 60, éste fue sustituido por el actual FASB.

Como se ha mencionado anteriormente, dos son los sistemas fundamentales para la preparación y presentación de la información contable: los sistemas basados en reglas y los basados en principios. Como indican Rodríguez y Bernad (2007):

Los modelos de regulación contable basados en reglas (rules-based) se caracterizan por que la información financiera está orientada al mero cumplimiento de las normas más que a proporcionar información para la toma de decisiones económicas. Por su parte, los modelos de regulación contable sustentados solo en principios (principles only) no proporcionan suficiente orientación y apoyo para la realización de juicios profesionales, de lo que resulta una aplicación dispar de las normas contables, con el consiguiente riesgo de pérdida de comparabilidad entre entidades.

Los US GAAP (normas basadas en reglas) han sido objeto de numerosas críticas, llegando a imputárseles un alto grado de responsabilidad en los grandes fraudes corporativos como el caso de Enron o World Com. La aprobación de la Ley Sabarnes-Oxley (2002) es muestra de la preocupación generada en este tema, tanto en cuanto, dicha ley contenía un mandato para que la SEC realizase un estudio sobre las implicaciones que tendría la adopción de un modelo de regulación contable basado en principios. Dicho estudio fue presentado en 
julio de 2003 "Study Pursuant 108(d) of the Sarbanes-Oxley Act of 2002 on the Adoption by the United States Financial Reporting System of a Principles-Based Accounting System" (RODRÍGUEZ; BERNAD, 2007).

Fruto de este estudio, la SEC recomendó que las normas contables se elaboraran siguiendo un modelo basado en principios que debería presentar las siguientes características (RODRÍGUEZ; BERNAD, 2007):

a) las normas deben estar basadas en un marco conceptual y aplicarlo consistentemente;

b) cada norma debe perseguir un objetivo claramente establecido;

c) cada norma debe suministrar suficiente orientación y apoyo para que pueda ser aplicada de modo uniforme;

d) las excepciones deben ser mínimas;

e) debe evitarse el uso de puntos de corte. Esto es, determinar el tratamiento contable de una operación en función de que se alcancen o no determinados niveles (normalmente, expresados en términos porcentuales) fijados de antemano.

Para asegurar qué se debe entender por presentar los estados contables de total conformidad con los US-GAAPS, la Declaración de Normas de Auditoría (Statement on Auditing Standards), SAS 69, establece lo que se denomina la jerarquía de los principios, delimitando cuatro categorías:

a) Categoría A - pronunciamientos de cuerpos autorizados (FASB, APB, CAP, SEC)

- FAS (Financial Accounting Standards) y FIN (FASB Interpretations), del FASB

- APB Opinions (Accounting Principles Board Opinions), Opiniones de la APB

- ARB (Accounting Research Bulletins) del Committeeon Accounting Procedure (CAP) del AICPA, Boletines de Investigación sobre Contabilidad

- La Regulation S-X y los Financial Reporting Releases de la SEC;

b) Categoría B - Pronunciamientos de cuerpos de expertos que se exponen para comentario público

- FTB (FASB Technical Bulletins), del FASB

- Industry Audit and Accounting Guides, del AICPA

- SOP (AICPA Statements of Position), del AICPA;

c) Categoría C - Pronunciamientos de cuerpos de expertos que no se han expuesto al comentario público

- Consensus Positions de la Emerging Issues Task Force del FASB

- PB, Practice Bullettins del AICPA;

d) Categoría D - Prácticas y pronunciamientos ampliamente aceptados, que representan la práctica habitual en una industria particular o las aplicaciones a circunstancias específicas

- AIN, Accounting Interpretations del AICPA

- FIG (Guías de Implementación del FASB)

- Prácticas de las industrias ampliamente reconocidas.

Además, para determinar el tratamiento contable de una operación no recogida en las normas anteriores, se debe analizar la posible aplicación (por orden de prioridad) de: i) los criterios contables que se aplicarían para transacciones o eventos similares según las categorías anteriores, y ii) otra literatura contable. Con la expresión otra literatura contable se hace referencia a otras fuentes, entre las que se encuentran los pronunciamientos conceptuales 
del FASB denominados Statements of Financial Accounting Concepts (CON), las normas e interpretaciones del IASB, los pronunciamientos de asociaciones profesionales o agencias reguladoras, libros de contabilidad y artículos (RODRÍGUEZ; BERNARD, 2007)

\section{LAS SUBVENCIONES EN LA NORMATIVA INTERNACIONAL - LA PARTICULARIDAD DE LOS DERECHOS DE EMISIÓN DE GEI}

El tratamiento contable de las subvenciones en la normativa internacional está regulado en la NIC 20 por parte del IASB, y la SFAS 116 por parte del FASB.

La NIC 20 se encuentra en estos momentos siendo objeto de una profunda revisión, que comenzó en julio de 2002, y que en la reunión de diciembre de 2007 fue de nuevo objeto de debate por su relación con el proyecto sobre Derechos de Emisión de Gases de Efecto Invernadero que el IASB tenía pendiente de estudio desde que en junio de 2005 retirara la interpretación IFRIC3 Emission Rights sin sustituirla por ninguna otra.

Con el objetivo de ver las implicaciones de esta situación, resumamos los puntos clave relacionados con dichos derechos. Desde la puesta en marcha del Esquema Europeo de Comercio de Derechos de Emisión de GEI tras la entrada en vigor del Protocolo de Kioto, y la entrada de dichos derechos como un nuevo elemento económico en el patrimonio de la empresas, las dudas sobre el registro contable de las operaciones relacionadas con ellos no se han solventado. En el caso de España, el ICAC optó por la publicación de la Resolución de 8 de febrero de 2006, amparándose en la ausencia de una guía internacional al respecto.

Dicha ausencia se debe a la retirada, antes mencionada, por parte del IASB de la Final Interpretation IFRIC 3, interpretación que pretendía ser la respuesta a todas las dudas sobre la contabilización de los Derechos de Emisión. Tal fue el rechazo entre asociaciones académicas y profesionales que incluso el EFRAG recomendó a la Unión Europea que no la adoptara dadas las distorsiones que provocaría en los Balances y Cuentas de Resultados de las empresas afectadas.

Los debates a nivel internacional sobre su correcta contabilización aun perduran, habida cuenta que el IASB ha pospuesto cualquier estudio al respecto hasta que no se pronuncie sobre los trabajos en curso relativos a la NIC 20 Subvenciones y la NIC 37 provisiones, NICs que a su vez afectan a los derechos de emisión

Las pautas que el IFRIC 3, antes mencionado, proponía eran las siguientes:

a) los derechos debían reflejarse como activos intangibles y ser reconocidos en los estados financieros de acuerdo a la NIC 38, tanto si son entregados por el gobierno como comprados;

b) cuando los derechos eran entregados a una empresa por el Gobierno del respectivo país (o por una agencia gubernamental) por debajo de su valor razonable, la diferencia entre la cantidad pagada, en su caso, y el valor razonable debía ser considerado como una subvención oficial que será contabilizada de acuerdo a la NIC 20;

c) los derechos quedan excluidos de ser calificados como existencias al considerarlos no inventariables, o como activos financieros, ya que no cumplen la definición de la NIC 32 para ser clasificados como tales. La única posibilidad sería modificar la NIC 39 para que los derechos pudieran clasificarse como disponible para la venta, ya que los derechos deben ser entregados al final de cada periodo del PNA en función de las emisiones realizadas;

d) aunque los derechos debían ser valorados inicialmente por su valor razonable, posteriormente era posible elegir entre un modelo de coste o un modelo de 
revalorización. En este último caso, las fluctuaciones en el valor de mercado de los derechos mantenidos debían ser reconocidas generalmente en el patrimonio de la empresa (a través del movimiento de las reservas), excluyéndose su traslado a la cuenta de Pérdidas y Ganancias;

e) dada la entrega gratuita de derechos por parte de una agencia gubernamental, estos deberán considerarse como una subvención, que debía ser periodificada como ingreso en el periodo en el que se mantuvieran los derechos;

f) a medida que una empresa producía emisiones, era necesario reconocer una provisión, dada la obligación de devolver los derechos de acuerdo a la NIC 37. Respecto a su cuantificación, la misma debía ser dotada por el importe coincidente con el valor de mercado de los derechos necesarios para justificar las emisiones realmente realizadas;

g) finalmente, se excluía la posibilidad de amortizar los derechos, en base a la ausencia de pérdida sistemática de valor, sin perjuicio de las correcciones por deterioro que le pudieran afectar.

Dado que los derechos asignados a cada instalación según el PNA vigente deben ser considerados como una subvención oficial, entra en juego la situación actual de revisión en la que se encuentra la NIC 20. Por ello es necesario un análisis de la misma. El objetivo de la revisión de esta norma es doble: por un lado eliminar las inconsistencias con el Marco Conceptual y por otro eliminar la diversidad de opciones que puedan reducir la comparabilidad entre estados financieros.

Respecto a las inconsistencias con el Marco Conceptual, se ha de señalar, como indica el IASB, que en el párrafo 12 cuando indica "Las subvenciones oficiales deben reconocerse como ingresos sobre una base sistemática, a lo largo de los ejercicios necesarios para compensarlas con los costes relacionados..." se da la circunstancia de que la empresa pueda estar reconociendo un crédito diferido cuando no ha reconocido ningún pasivo.

Además, se ha de tener en cuenta que la actual redacción de la NIC 20 también es inconsistente con los pronunciamientos más recientes que otros organismos han hecho sobre esta materia, como es el caso de la SFAB 116 Accounting for Contributions Received and Contributions Made, la UIG 11 Accounting for contributions of, or contributions for the adquisition of, non-current assets y el IPSAS 23 Revenue from Non-Excahnge Transactions. De hecho, la adaptación del ICAC en nuestro PGC2007 de la IAS20 elimina la inconsistencia antes mencionada (CIUDAD; MILANÉS, 2009).

Respecto a la diversidad de opciones, destaca la posibilidad de, en el caso de subvenciones no monetarias, reconocer por un valor simbólico tanto la subvención como los activos recibidos, aun cuando la norma general debiera ser el uso del valor razonable.

Las primeras revisiones de la NIC 20 comienzan en la discusión de julio de 2002 del IASB. Partiendo de la base de que la norma en ese momento había quedado desfasada, se plantearon cinco alternativas: no hacer nada, retirar la IAS20, converger hacia la SFAS 116, converger hacia la UIG 11, o desarrollar una nueva norma.

No es hasta la reunión de febrero de 2004 que el IASB recomienda la retirada de la norma, frente a la alternativa propuesta de modificarla aplicándole los modelos contenidos en la NIC 41 sobre Activos Biológicos.

En febrero de 2006, se decide aplazar los trabajos en curso relacionados con la NIC 20 hasta que la NIC 37 no sea revisada, lo que lleva a una nueva discusión en diciembre de 2007 cuando se activa el proyecto de estudio de los Derechos de Emisión de Gases de Efecto Invernadero que se ve afectado por lo que se concluya respecto esas dos NIC mencionadas. 
Así, se decide limitar la revisión de la NIC 20 a los términos que afectan al comercio de dichos derechos, y aplazar la revisión global de la norma.

Dada la retirada en junio de 2005 de la que pretendía ser la guía internacional en materia de Derechos de Emisión, y dada la dilación en el tiempo de la revisión de las normas implicadas (directa o indirectamente) en las operaciones relativas a este nuevo instrumento patrimonial, muchas empresas han decidido aplicar la NIC 8, lo que ha provocado una diversidad de tratamientos contables cuanto menos sorprendentes.

Ello a su vez ha provocado situaciones de incomparabilidad contable nada recomendables. Algunas empresas han optado por reconocer los derechos asignados bien por su valor de cotización del día de la asignación o a valor cero, o los han clasificado con inmovilizados intangibles, como existencias, o como activos financieros, se han amortizado en algunos casos y en otros no y así, hasta 15 alternativas según se desprende del informe elaborado por PWC.

En efecto, la NIC 8 de Políticas contables, cambios en las estimaciones contables y errores. La citada Norma Internacional, en su punto 10, indica que "En ausencia de una Norma o Interpretación que sea aplicable específicamente a una transacción, otros hechos o condiciones, la dirección deberá usar su juicio en el desarrollo y aplicación de una política contable " y en el punto 12 se señala que al realizar los juicios descritos en el párrafo 10, la dirección podrá considerar también los pronunciamientos más recientes de otros instituciones emisoras de normas, que empleen un marco conceptual similar al emitir normas contables.

Ello ha dado pie para que algunas empresas europeas hayan decidido seguir las indicaciones de la SFAS 116 de la normativa norteamericana para registrar las operaciones relacionadas con los Derechos de Emisión asignados.

\section{LOS CONTACTOS IASB Y FASB - SITUACIÓN ACTUAL DEL DEBATE}

Como se ha comentado anteriormente, el IASB centró la reunión de febrero de 2006 en la situación de la NIC 20 sobre subvenciones con el objeto de revisarla. Incluso algunos miembros del Board consideraron que lo mejor que se podía hacer era retirarla. Si bien la mayoría decidió que ello sería dar un paso en la dirección correcta, pensaron que el vacío que dejaría en el tema de Subvenciones no sería nada deseable.

Además, se indicó que ciertas operaciones sobre reconocimiento y cuantificación de obligaciones relacionadas con subvenciones eran similares a temas de reconocimiento y cuantificación de provisiones recogidas en la NIC 37. Dado que en esos momentos el IASB estaba reconsiderando la NIC 37 como parte de su proyecto sobre Combinación de Negocios, se decidió posponer los trabajos sobre la NIC 20 a la espera de las decisiones finales sobre la NIC 37.

Por tanto, hasta que ambas NIC no sean estudiadas por el IASB, la guía sobre Derechos de Emisión quedaría pendiente. Las inconsistencias de la NIC 20 por un lado, y el uso de las SFAS 116 por parte de empresas no norteamericanas dada la falta de guía internacional en materia de Derechos de Emisión hacen necesario por una parte profundizar en el proceso de convergencia internacional ya comentado, y por otra, insistir en que el IASB retome cuanto antes el proyecto - ahora conjunto con el FASB - sobre materia de Derechos de Emisión.

En este sentido, tras la última reunión de IASB y el FASB en octubre de 2008 relativa a dicho proyecto conjunto sobre los Esquemas de Comercio de los Derechos de Emisión, si bien se ha seguido posponiendo la emisión de una guía completa en la que se discuta un modelo de contabilidad para estos derechos, se ha alcanzado cierto consenso en algunos aspectos previos. 
Los miembros de ambos Boards ven la necesidad de emitir una guía que contemple los temas de reconocimiento de activos, valoración y deterioro, reconocimiento de pasivos y su valoración, momento de reconocimiento en pérdidas y ganancias, contabilización de derechos dependiendo de su año de emisión (añadas) y la presentación en los Estados Contables.

Así mismo, es intención de este proyecto que abarque todo tipo de derechos negociables y esquemas de negociación posibles. Es decir, debe cubrir tanto los esquemas basados en un sistema cap and trade mayoritarios en Europa, como los de baseline and credit, los créditos obtenidos a través de proyectos de Mecanismo de Desarrollo Limpio (los conocidos como CER's) e incluso los certificados obtenidos por el uso de energías renovables en empresas del sector eléctrico.

En un sistema cap and trade la autoridad competente asigna los derechos entre los participantes, que pueden ser negociados en un mercado. Al final de periodo de cumplimiento, los participantes están obligados a devolver a dicha autoridad el numero de derechos que iguala su emisión de gases contaminantes, debiendo pagar una multa si emiten por encima de los derechos que entreguen. En un sistema baseline and credit cada participante tiene asignado un límite por periodo. Al finalizar dicho periodo, las emisiones se comparan con el límite. Si se ha emitido menos, se reciben créditos negociables por la diferencia. Si se ha emitido más, la empresa debe comprar los créditos necesarios y remitirlos a la autoridad correspondiente.

Por último, el modelo debe abarcar tanto a los participantes de estos Esquemas por ser emisores de Gases de Efecto Invernadero, como al resto de agentes que deseen participar en él simplemente como compradores o vendedores de derechos negociables en un mercado organizado.

Según las agendas de estos comités, parece probable que para finales de 2009 dispongamos ya de la deseada guía internacional para la contabilización de los Derechos de Emisión en sentido amplio, lo que a su vez dará pie a una unificación de criterios sobre la mejor alternativa para recoger el impacto de una subvención no monetaria (con valor razonable) en el Patrimonio de la empresa y en su Cuenta de Resultados.

En esta línea, se ha de recordar la no objeción por parte del FASB de incluir los derechos de emisión dentro del proyecto de revisión del ARB n ${ }^{\circ} .43$ Restatement and Revision of Accounting Research Bulletins. Este proyecto supone el uso del valor razonable para ciertas formas de activos físicos no financieros negociables (commodities)

\section{CONCLUSIONES}

Es indudable la necesidad de una información financiera comparable a nivel internacional en un mercado de capitales global. Dicha información debe ser elaborada bajo unos estándares de alta calidad para que cumplan su objetivo, siendo la participación del IASB y el FASB clave en dicha elaboración.

La evolución de los sistemas contables internacionales se ha basado en la idea de conseguir un único cuerpo normativo de alta calidad tanto para la elaboración de los estados contables domésticos como transnacionales y es esta dirección en la que actualmente están trabajando ambos emisores de normas.

Desde los acuerdos de Norwalk en 2002, FASB e IASB están coordinando sus agendas de trabajo y desarrollando proyectos conjuntos que pongan las bases para esos estándares de alta calidad únicos. La actualización en septiembre de 2008 del Memorandum of Undertanding, firmado en 2006 , es una prueba de que los trabajos de convergencia siguen adelante.

Como señala en el documento elaborado por el FASB a requerimiento de la SEC en marzo de 2009 (http://www.fasb.org), la actual crisis financiera internacional global ha 
revelado la debilidad de un sistema internacional compuesto por múltiples conjuntos de normas contables, lo que hace todavía mas necesario el desarrollo de un lenguaje contable único.

En particular, y respecto al tema que nos ocupa, se necesita una revisión en profundidad de la NIC 20 sobre Subvenciones para eliminar las inconsistencias actuales con el Marco Conceptual y así unificar criterios para conseguir que las empresas que participan en los diferentes Esquemas de Comercio de Derechos de Emisión elaboren estados financieros comparables y útiles en los procesos de decisión empresarial.

\section{REFERENCIAS}

CIUDAD, A.; MILANÉS, P. Tratamiento contable de las subvenciones en el nuevo marco normativo contable español: opciones de políticas contables y tendencias de futuro. Técnica Contable n' 717, 2009.

COMISIÓN AL CONSEJO Y AL PARLAMENTO EUROPEO. La estrategia de la Unión Europea en materia de información financiera: el camino a seguir. Comunicación de la Comisión al Comisión al Consejo y al Parlamento Europeo COM(2000) 359 final, Bruselas, 13 de junio de 2000.

COMISIÓN EUROPEA. Armonización contable: una nueva estrategia de cara a la armonización internacional, noviembre de 1995.

EUROPEAN FINANCIAL REPORTING ADVISORY GROUP (EFRAG). Disponible en: <http://www.efrag.org> . Acceso en: 2009.

FINANCIAL ACCOUNTING STANDARD BOARD (FASB). Disponible en: $<$ http://www.fasb.org/FAF_SEC_Roadmap_Response_Final_with_Appendix.pdf $>$. Acceso en: 2009.

GARCÍA-AYUSO COVARSÍ, M. Reflexiones sobre el proceso de Armonización Internacional de la Contabilidad. Congreso Nacional de Auditoría Instituto de Censores Jurados de Cuentas de España, 15., Bilbao, 2006.

GINER INCHAUSTI, B. Algunas claves sobre la contabilidad europea: el nuevo proceso regulador y las nuevas normas. Estabilidad Financiera, n. 5, p. 53 y ss, 2004.

INTERNATIONAL ACCOUNTING STANDARD BOARD (IASB). Disponible en: <http://www.iasplus.com/standard/standard.htm>. Acceso en: 2009.

PRICEWATERHOUSECOOPERS AND IETA. Trouble - entry accounting. Revisited. uncertainty in accounting for the EU Emissions Trading Scheme and Certified Emission Reductions, 2007. Disponible en: <http://www.ukmediacentre.pwc.com/imagelibrary/ detail.asp?MediaDetailsID=899>. Acceso en: 2009.

RODRÍGUEZ, C.J.; BERNAD, A. Algunas cuestiones relevantes en el proceso internacional de convergencia contable: IASB versus FASB. Estabilidad Financiera, n. 13, 2007.

SECURITIES AND EXCHANGE COMMISSION (SEC). Report on Promoting Global Preeminence of American Securities Markets. October 1997. Disponible en: <http://www.sec.gov/news/studies/acctgsp.htm>. Acceso en: 2009.

SECURITIES AND EXCHANGE COMISIÓN (SEC). Proposed Rule 33-8818: Acceptance from Foreign Private Issuers of Financial Statements Preparedin Accordance with International Financial Reporting Standards without Reconciliation to U.S. GAAP. 2 de julio de 2007. 
STREET, D. L. An interview with Sir David Tweedie, Chair International Accounting Standards Board. Journal of International Financial Management and Accounting, n. 13, p. 73-100, 2002. doi:10.1111/1467-646X.00079

US-GAAP. La declaración de normas de auditoría. Statement on Auditing Standards SAS 69. Disponible en: 〈http://www.aicpa.org>. Acceso en: 2009. 